\title{
Regional and supraregional biochemistry services in Scotland: a survey of hospital laboratory users
}

\author{
M J Murphy, F J Dryburgh, J Shepherd
}

\begin{abstract}
Aim-To ascertain the views of Scottish hospital laboratory users on aspects of regional and supraregional biochemical services offered by the Institute of Biochemistry at Glasgow Royal Infirmary. Methods-A questionnaire was circulated asking questions or inviting opinions under various headings, including current patterns of usage of the services provided, availability of information on specimen collection requirements and reference ranges, current arrangements for transport of specimens, turnround times for delivery of reports, layout and content of request and report forms, quantity and quality of interpretive advice, potential changes in laboratory services, and overall impression of the services provided. Opportunities were provided for free text comment. The questionnaire was circulated in 1992 to heads of department in 23 Scottish hospital biochemistry laboratories.
\end{abstract}

Results-Twenty one replies were received. Services used widely included trace metals/vitamins $(n=20)$ and specialised endocrine tests $(n=19)$. Other services also used included specialised lipid tests $(n=13)$, toxicology $(n=12)$, thyroid function tests $(n=$ nine $)$, and tumour markers ( $n=$ eight). Fifteen laboratories used one or more of the services at least weekly. Most $(n=20)$ welcomed the idea of a handbook providing information on specimen collection and reference ranges. Nine identified loss of specimens as a problem. Other perceived problems included the absence of reference ranges from report forms, quantity and quality of interpretive advice, and turnround times of some tests. Overall impressions of the service(s) offered were very $\operatorname{good}(n=12)$; adequate ( $n=$ seven); poor $(\mathbf{n}=$ one).

Institute of Biochemistry, Glasgow Royal Infirmary M J Murphy F J Dryburgh J Shepherd

Correspondence to: Dr M J Murphy, Institute of Biochemistry, Royal G4 0SF.

Accepted for publication

3 November 1993
Conclusions-Useful information was obtained about patterns of use and transport arrangements. Areas identified as requiring follow up included provision of information, alternative ways of communicating reports, and improvement in quantity and quality of interpretive advice.

(F Clin Pathol 1994;47:395-398)
Regional and supraregional assay services comprise an important part of the investigation of hospital inpatients, particularly those who are subjects of postprimary referral, as well as many patients seen by general practitioners. The results of such investigations are often critical to decisions about patient management, and therefore the availability and quality of these services assume considerable importance. In Scotland, funding for regional and supraregional assay services comes partly from the Scottish Hospitals' Allocation for Resource Expenditure (SHARE) formula, and partly from crosscharging (in the case of hospitals that have achieved full trust status). The benefits of centralising these services and of ensuring that appropriate future arrangements are made in the context of NHS reforms have been recognised, ${ }^{12}$ but there has not previously been any formal appraisal within the United Kingdom of the quality of the services provided. We sought to obtain information and opinions from users of the regional and supraregional assay services provided by our own department. We did not seek to assess similar services provided by other laboratories.

Virtually all specimens that require analysis outwith local hospital laboratories are nevertheless initially sent to and separated by the local laboratory, which arranges for their subsequent dispatch. Also, reports from the referral laboratory are returned to the local laboratory, which may then choose to issue its own reports and interpretive comments. (Referral of specimens and reports through the local laboratory permits efficient use of transport personnel and resources; occasionally, it may be appropriate to communicate directly with clinicians.) It therefore seemed appropriate to survey hospital biochemistry laboratories to achieve our objectives.

(Note: our laboratory carried out 2.4 million tests in 1992. Twenty seven per cent of its resources-weighted laboratory workload-was devoted to tests from other laboratories. The repertoire of tests and the workload of referring laboratories varied widely.)

\section{Methods}

A survey of hospital laboratory users of the regional and supraregional assay services offered (see appendix 1) by the Institute of 
Biochemistry at Glasgow Royal Infirmary was carried out as part of a departmental audit programme. Questionnaires were sent to all 23 heads of department in biochemistry laboratories in Scottish hospitals, and 21 replies were received. Information and views were sought on aspects of specimen collection and transport, request and report forms, delivery of reports, availability and quality of interpretive advice, and potential changes in laboratory services. Those surveyed were also asked for their overall impression of the services provided. The questionnaire consisted of a combination of "closed" questions (yes/no replies or ticking of options) and several opportunities for free text comment. Matrices were constructed to allow flexibility in commenting on different sections of the service. Respondents' free text comments were summarised and categorised separately from replies to closed questions.

\section{Results}

Twenty one of 23 heads of department in Scottish hospital biochemistry laboratories replied to the questionnaire.

GENERAL

Those sections most widely used were trace metals/vitamins $(n=20)$, gonadal $(n=17)$, adrenal $(n=19)$, and other specialised endocrine tests $(n=19)$. Other sections widely used included specialised lipid tests $(n=13)$, toxicology ( $n=12)$, thyroid function tests $(\mathrm{n}=$ nine), and tumour markers ( $n=$ eight $)$.

\section{INFORMATION ON SAMPLE COLLECTION AND} REFERENCE RANGES

Views were sought on the information available to users on specimen collection and reference ranges. Some considered that the information available on some sections was non-existent or poor-namely, adrenal ( $n=$ three), other endocrine ( $n=$ one), toxicology ( $\mathrm{n}=$ three $), \quad$ lipids $\quad(\mathrm{n}=$ seven $), \quad$ trace metals/vitamins ( $n=$ three), and tumour markers $(n=t w o$ ). All but one said that they would find a handbook containing this information useful. Respondents were asked to specify any other information, on specimen collection and reference ranges, that they would see as being especially useful. Aspects mentioned included specimen requirements ( $n=$ five), turnround times of tests $(n=$ five),

Table 1 Do the turnround times for the following results meet the needs of your requesting clinicians?

\begin{tabular}{lrl}
\hline & Yes & No \\
\hline Thyroid & 7 & 1 \\
Gonadal & 11 & 5 \\
Adrenal & 13 & 5 \\
Other endocrine & 12 & 5 \\
Toxicology & 7 & 2 \\
Lipids & 8 & 3 \\
Trace metals/vitamins & 17 & 0 \\
Tumour markers & 6 & 1 \\
Other & 2 & 1 \\
\hline
\end{tabular}

regular updates of information $(n=$ two) as well as quality control data, projected charges for the coming year, range of tests available, and stability of analytes $(n=$ one each).

\section{PROCESSING AND TRANSPORT OF SPECIMENS}

Nearly all the laboratories surveyed $(n=19)$ spin down specimens before sending them on (assuming that whole blood is not required), and most $(n=16)$ complete a separate request form for their own records. Only five ever add clinical information if they consider that this is warranted.

Fifteen laboratories send specimens on a weekly basis at least, and of these, seven send on a daily basis. Twelve use the Royal Mail for transport of specimens, 10 private transport and personnel, and seven NHS transport and personnel. Similar numbers send specimens individually $(n=10)$ or in batches $(n=13)$. When asked about loss of specimens, 18 perceived the problem as occurring at the level of processing of specimens within Glasgow Royal Infirmary or return of reports to the local hospital.

\section{DELIVERY OF REPORTS}

Some sections were seen to perform better than others with respect to turnround times (table 1). Twelve cited endocrine tests as examples of where there was a failure to meet clinicians' needs. Sixteen said that they would find a direct line to biochemistry, bypassing the switchboard useful; however, only eight thought that faxing of results would help.

\section{REPORTS AND INTERPRETIVE ADVICE}

Nine respondents considered that the layout and content of report forms (for an example, see appendix 2) could be improved. When asked to specify how, six cited the inclusion of reference ranges for specialised endocrine tests. Small numbers (three or less) cited other sections of report forms, such as results, comments, patient identification data (PID) information, and clinical information.

Although few (two or less) considered that interpretive advice was rarely supplied, significant numbers thought that it was supplied only intermittently (table 2). Most comments focused on the absence of informative comment or advice on further investigations. When asked whether they could make use of the names and extension numbers of individual persons within the laboratory for specialist advice, all of those who replied $(n=18)$ said yes.

Table 2 Do we provide the clinical/interpretive advice that you need for the following groups of test?

\begin{tabular}{lcll}
\hline & Generally & Intermittently & Rarely \\
\hline Thyroid & 6 & 2 & 2 \\
Gonadal & 9 & 5 & 2 \\
Adrenal & 11 & 6 & 2 \\
Other endocrine & 11 & 4 & 2 \\
Toxicology & 4 & 4 & 0 \\
Lipids & 3 & 6 & 1 \\
Trace metals/vitamins & 9 & 6 & 1 \\
Tumour markers & 5 & 1 & 1 \\
Other & 1 & 0 & 0 \\
\hline
\end{tabular}


Table 3 What is your overall impression of the service we offer you?

\begin{tabular}{lr}
\hline Very unsatisfactory service & 0 \\
Poor service & 1 \\
Adequate service & 8 \\
Very good service & 12 \\
\hline
\end{tabular}

\section{POTENTIAL CHANGES}

Four respondents indicated that there were tests not currently offered which they would like: thyroglobulin $(n=2), \operatorname{LSD}(n=1)$, and a wider range of gut hormones $(n=1)$.

\section{OVERALL IMPRESSION}

Table 3 shows the answers to the question, "what is your overall impression of the service we offer you?" Four respondents qualified their opinion in some way-for example, adequate (barely), very good (on the whole), etc. Several included critical but constructive statements in the space provided for free text comment. These centred once again on the quantity and quality of interpretive comment, on turnround times, and on loss of specimens or reports.

\section{Discussion}

Twenty one out of 23 questionnaires were completed and returned; thus the information and opinions obtained in the survey are representative of users of Scottish hospital biochemistry laboratories, and an accurate reflection of current patterns of usage and practice. The patterns of use found are not surprising themselves: they merely reflect the balance between "core analysis" services-the routine analyses carried out locally-and regional/supraregional assay services. This is, however, a shifting balance: the advantages of centralising specialised analytical work (potentially more efficient and economical use of resources and personnel) must be weighed against the disadvantages (potentially increased turnround times and specimen loss rates). The advent of hospital trusts has focused attention on this aspect of resource allocation more than ever, and for local laboratories the decision on whether or not to refer samples for analysis depends critically on the quality of the service provided. Hence the need for laboratories providing regional and supraregional services to assess regularly the perception of the quality of these services.

We identified a major need on the part of hospital laboratory users to have improved access to more comprehensive information on the range of tests available, on specimen collection requirements, on reference ranges, on turnround times, on quality control data, and on projected charges for tests. This echoes a similar finding in a previous survey of general practitioners. $^{3}$ The provision of a laboratory handbook containing such information, supplemented as necessary by updates, is an obvious way of meeting this need.

Only five out of 21 laboratories ever add clinical information to request forms if they consider that it is warranted. This is an area in which improved cooperation could certainly improve the quality of the services provided.

Although the rate of specimen loss in our laboratory has not been formally assessed, it probably does not exceed $1 \%$. This belies the high profile that attaches to this problem. Eighteen of 21 respondents identified the problem with loss of specimens as occurring at the level of processing of specimens within Glasgow Royal Infirmary, or return of reports to the local hospital, rather than transport of specimens to Glasgow Royal Infirmary; this transport is the only step in the process over which users have direct control. It is clearly impossible to eradicate completely the problem of specimen loss, short of a chain of custody such as is used with forensic specimens; however, the acceptability or otherwise of a particular loss rate is at least partly determined by the resources available to reduce it.

Loss of specimens is readily documented. Assessing the quality of interpretive advice and attempting to improve it is considerably more difficult. Reporting biochemists in general rarely have access to the full clinical picture and are dependent on the information supplied by the requesting clinicians. Although in principle it is possible to establish guidelines both for clinicians, in the supply of information, and for biochemists, in the provision of informed comment, in practice such guidelines are not followed often and comments are usually generated as free text. Problems with communication are best tackled by providing easier access to laboratory staff: all of those who replied indicated they could make use of the names and extension numbers of people within the department. Similarly, the concept of an identifiable duty biochemist, often with responsibility for a particular area, was welcomed.

The reporting practices of different specialised sections are based on several factors, including the throughput of specimens and the perceived importance of results for patient management. Routine telephoning of all abnormal results is not always feasible. When the throughput of specimens is small and the perceived penalty to the patient for failing to do so is serious-for example, tumour markers-the decision to phone results is easy; for other, more heavily used sections, such a policy is impractical; selection of results for telephoning is at the discretion of the reporting biochemist in the referral laboratory.

In conclusion, virtually all of the problems identified in this survey relate to communication. There is a need on the part of users for more information in some areas, most notably specimen collection requirements and reference ranges; and where it is not feasible to provide reference ranges on report forms (for instance, some endocrine tests), this fact should be communicated to users. Likewise, solutions to the problem of turnround times include pursuing alternative ways of communicating results - for example, faxing, direct computer linkups and keeping users informed about the frequency with which tests are performed. Advice from specialised sections is 
often as important as the quality of the analytical phase. Improving the quantity and quality of interpretive advice is likely to require considerable effort on the part of everyone to communicate more effectively: a survey is merely the first step in this direction. Since this survey was completed, a direct line to the biochemistry reporting office has been installed, bypassing the switchboard; a handbook has been produced containing much of the information desired by the users of our services; and certain request forms are being redesigned. It is hoped that exchanges of views and information will continue.

1 Lester E. The supraregional assay service. $B M \mathcal{F} 1992 ; 304$ 790-1.

2 Steward JC, Oakey RE. Future arrangements for the supraregional assay service. Bulletin of the Royal College of Pathologists 1991;74:10-11.

3 Paterson JR, Murphy MJ, Langan J, Dryburgh FJ, Shepherd J. "What do you think of the service?" An Shepherd J. "Wh audit of local general practitioner requirements. Bulletin of the Royal College of Pathologists 1992;77:15-16.

\begin{tabular}{|c|c|}
\hline $\begin{array}{l}\text { Thyroid } \\
\text { Gonadal } \\
\text { Adrenal }\end{array}$ & $\begin{array}{l}\text { T4, T3, TSH, TBG, FT4 } \\
\text { LH, FSH, prolactin, oestradiol, testosterone, progesterone, SHBG } \\
\text { Cortisol, ACTH, 17-hydroxyprogesterone, dehydroepiandrosterone sulphate, } \\
\text { androstenedione, aldosterone }\end{array}$ \\
\hline Other endocrine & $\begin{array}{l}\text { GH, insulin, C-peptide, gastrin, pancreatic polypeptide, glucagon, PTH, } \\
\text { calcitonin, 25-hydroxy vitamin D, 1,25-dihydroxy vitamin D, cAMP, IGF-1 }\end{array}$ \\
\hline Toxicology & $\begin{array}{l}\text { Salicylate, paracetamol, phenytoin, phenobarbitone, primidone, } \\
\text { carbamazepine, valproate, theophylline, digoxin, methotrexate, cyclosporine, } \\
\text { drugs of abuse }\end{array}$ \\
\hline Lipids & $\begin{array}{l}\text { Total cholesterol, triglycerides, VLDL, LDL, HDL, apoprotein A-1, apoprotein B, } \\
\text { lipoprotein (a) }\end{array}$ \\
\hline Trace metals & $\begin{array}{l}\text { Magnesium, zinc, copper, iron, selenium, aluminium, cadmium, lithium, lead, } \\
\text { mercury }\end{array}$ \\
\hline $\begin{array}{l}\text { Vitamins } \\
\text { Tumour markers }\end{array}$ & $\begin{array}{l}\text { A,B-1, B-2, B-6, B-12, C,E, folate } \\
\text { AFP, hCG, CEA, ACP, PSA }\end{array}$ \\
\hline
\end{tabular}

Appendix 2

\section{BIOCHEMISTRY DEPARTMENT}

GLASGOW ROYAL INFIRMARY

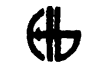

E5

Name

Address

Address

Consultant

D.o.b.

Hospital

Ward

Hospital Number

Date sampled

Date received

Accession Number

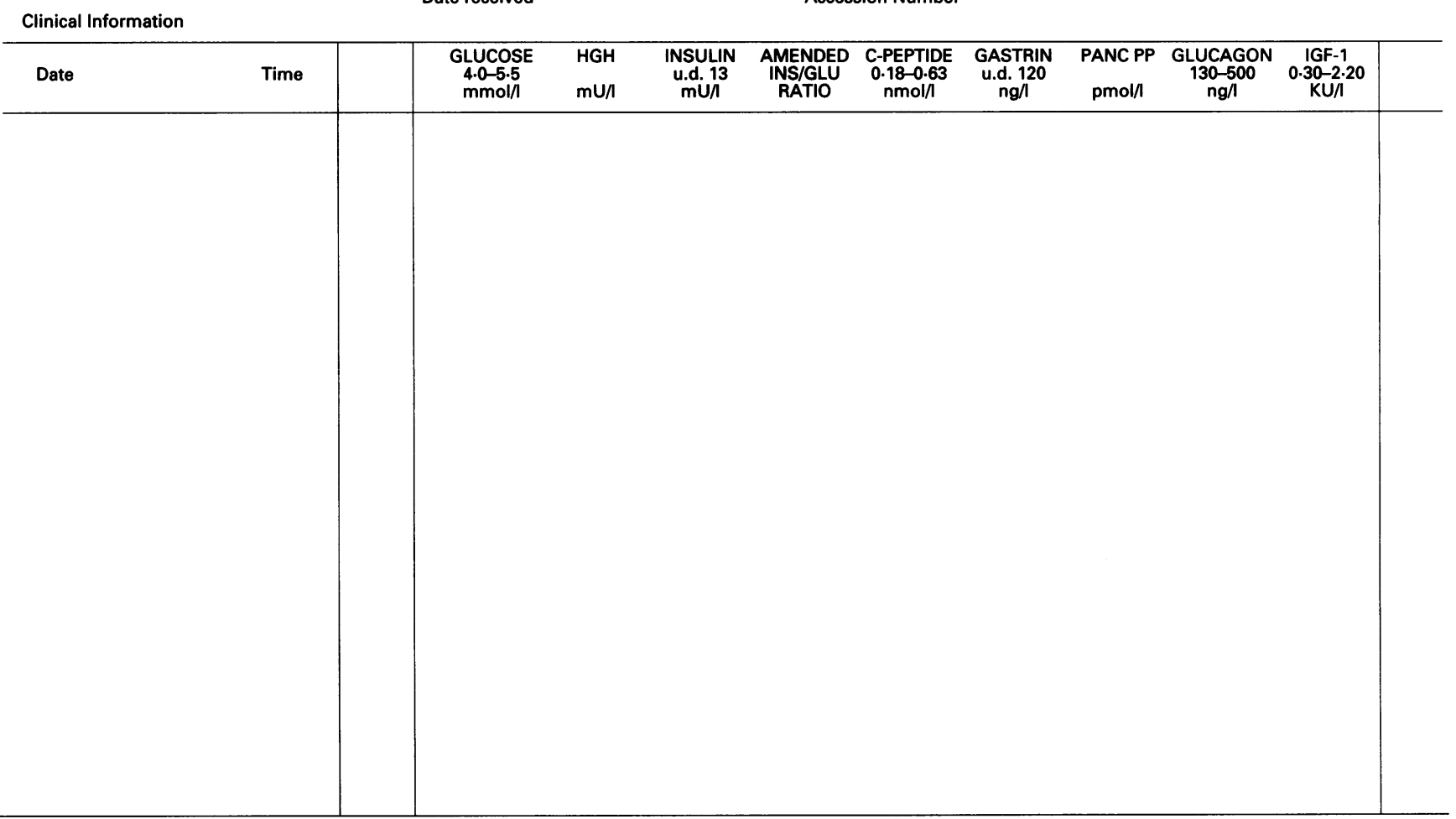

